\title{
Lentiviral Interleukin-10 Gene Therapy Preserves Fine Motor Circuitry and Function After a Cervical Spinal Cord Injury in Male and Female Mice
}

\author{
Jessica Y. Chen ${ }^{1,2}$ • Emily J. Fu ${ }^{1}$ Paras R. Patel ${ }^{1}$ - Alexander J. Hostetler ${ }^{1} \cdot$ Hasan A. Sawan ${ }^{1} \cdot$ Kayla A. Moss ${ }^{1}$. \\ Sarah E. Hocevar ${ }^{1,2} \cdot$ Aileen J. Anderson ${ }^{3} \cdot$ Cynthia A. Chestek ${ }^{1,2,4,5} \cdot$ Lonnie D. Shea $^{1,2}$ (1)
}

Accepted: 6 October 2020 / Published online: 13 October 2020

(C) The American Society for Experimental NeuroTherapeutics, Inc. 2020

\begin{abstract}
In mammals, spinal cord injuries often result in muscle paralysis through the apoptosis of lower motor neurons and denervation of neuromuscular junctions. Previous research shows that the inflammatory response to a spinal cord injury can cause additional tissue damage after the initial trauma. To modulate this inflammatory response, we delivered lentiviral anti-inflammatory interleukin-10, via loading onto an implantable biomaterial scaffold, into a left-sided hemisection at the C5 vertebra in mice. We hypothesized that improved behavioral outcomes associated with anti-inflammatory treatment are due to the sparing of fine motor circuit components. We examined behavioral recovery using a ladder beam, tissue sparing using histology, and electromyogram recordings using intraspinal optogenetic stimulation at 2 weeks post-injury. Ladder beam analysis shows interleukin10 treatment results in significant improvement of behavioral recovery at 2 and 12 weeks post-injury when compared to mice treated with a control virus. Histology shows interleukin-10 results in greater numbers of lower motor neurons, axons, and muscle innervation at 2 weeks post-injury. Furthermore, electromyogram recordings suggest that interleukin-10-treated animals have signal-to-noise ratios and peak-to-peak amplitudes more similar to that of uninjured controls than to that of control injured animals at 2 weeks post-injury. These data show that gene therapy using anti-inflammatory interleukin-10 can significantly reduce tissue damage and subsequent motor deficits after a spinal cord injury. Together, these results suggest that early modulation of the injury response can preserve muscle function with long-lasting benefits.
\end{abstract}

Key Words Gene therapy $\cdot$ biomaterial $\cdot$ spinal cord injury $\cdot$ inflammation $\cdot$ neuromuscular junction

Emily J. Fu and Paras R. Patel contributed equally to this work.

Electronic supplementary material The online version of this article (https://doi.org/10.1007/s13311-020-00946-y) contains supplementary material, which is available to authorized users.

Lonnie D. Shea

ldshea@umich.edu

1 Department of Biomedical Engineering, College of Engineering, University of Michigan, 2200 Bonisteel Boulevard, 1119 Carl A Gerstacker Building, Ann Arbor, MI 48109, USA

2 Neuroscience Graduate Program, University of Michigan Medical School, Ann Arbor, MI 48109, USA

3 Department of Anatomy and Neurobiology, University of California, Irvine, CA 92697, USA

4 Department of Electrical Engineering and Computer Science, University of Michigan, Ann Arbor, MI 48109, USA

5 Robotics Program, University of Michigan, Ann Arbor, MI 48109. USA

\section{Introduction}

The loss of complex neural connections and inadequate nerve growth after a spinal cord injury (SCI) often results in permanent motor deficits or even complete paralysis [1]. Trauma can result in the apoptosis of lower motor neurons (LMNs), whose axons become detached from neuromuscular junctions (NMJs), leaving behind denervated motor endplates [2]. With sustained loss of neural input, these orphaned motor endplates will then release chemotactic signals to induce nearby, innervated NMJ axons to sprout growth cones [3,4]. An orphaned motor endplate may then become reinnervated to form a functional NMJ. Alternatively, if the motor endplate remains denervated, it will disintegrate, the muscle fiber will atrophy, and paralysis will remain [5-9]. 
The early inflammatory response following trauma to the spinal cord can cause additional damage to neural tissues beyond the initial mechanical trauma $[10,11]$. Immediately after the blood-brain barrier is compromised, peripheral monocytes infiltrate and differentiate into macrophages that can exhibit phenotypes ranging from pro-inflammatory to anti-inflammatory. An inflammatory phenotype is highly linked to the secondary damage seen with an SCI, whereas the antiinflammatory phenotype has been shown to encourage cell survival and tissue regeneration [12, 13]. Interleukin-10 (IL10) is an anti-inflammatory cytokine that both is expressed by macrophages and induces macrophage polarization towards an anti-inflammatory phenotype, which in turn results in a downregulation of pro-apoptotic factors and an upregulation in anti-apoptotic factors [14-17]. In the absence of IL10, secondary damage is exacerbated [18]. Thus, shifting the postinjury response towards an anti-inflammatory phenotype has been a major target for therapeutic intervention.

The neuroprotective effects of IL10 treatment for an SCI have been investigated in several rodent studies. IL10 provides trophic support directly to neurons through the IL10 receptor [19], decreases post-injury inflammation [20], and increases myelination after a cervical SCI [21]. Many have shown that IL10 decreases lesion volume and apoptosis, while increasing behavioral recovery [20, 22-29]. However, the majority of animal SCI studies are carried out in female subjects [22-27, 29, 30], while most human SCIs occur in males [31]. Significant sex differences have been observed in immune responses after an SCI [32-34]. Furthermore, the majority of animal SCI studies are carried out at the thoracic level [23-29], while cervical level injuries are estimated to make up about half of all human SCIs [35]. In contrast to thoracic spinal tissues, the cervical enlargement contains a greater proportion of circuitry that is responsible for fine motor control, and the impact of IL10 treatment in a cervical SCI has not been investigated.

In this study, we examined immunomodulation after a cervical SCI and assessed the sparing of fine motor circuitry and functional recovery, using both male and female mice. A left C5 lateral hemisection was performed, resulting in loss of function of the left arm. We chose to test a penetrating injury as individuals with penetrating SCI exhibit little improvement, if any, over time. The penetrating injury was created by tissue resection to yield a consistent injury. The resected tissue was replaced with a biomaterial bridge made of poly(lactide-coglycolide) (PLG), which clearly delineates the location of the injury. This implant allows for precise analyses of peri-injury tissues, where secondary damage can be examined without interference from the primary injury. The PLG bridge is implanted after loading with a lentivirus encoding for IL10, with lentiviral firefly luciferase (FLuc) serving as a control. Lentivirus delivery has previously been shown to induce localized and sustained transgene expression [36]. Containing an anti-inflammatory gene therapy to the injury location is advantageous because then the consequences of systemic immunosuppression can be avoided, while sustained transgene expression is necessary because post-SCI inflammation can persist for multiple weeks. Our lab has previously verified that lentiviral IL10 expression can shift post-SCI gene expression towards an anti-inflammatory profile $[37,38]$.

Herein, we examined behavioral recovery using a ladder beam at 2 and 12 weeks post-injury (wpi). Then, using immunohistochemistry, we quantified LMNs, axons, motor endplate densities, innervated NMJ densities, fraction of total NMJs that are innervated, and growth cone sprouting. We targeted the acromiotrapezius (ATZ) due to its topographical representation rostrally and throughout $\mathrm{C} 5$. In addition, we analyzed the flexor digitorum profundus and carpi ulnaris (FLX) together, due to their small size and topographical representation beginning in caudal C5 [39]. Finally, optogenetic stimulation was used to examine muscle activatability, which was quantified as a signal-to-noise ratio (SNR) and peak-topeak (P2P) amplitude on an electromyogram (EMG). Our results show that early therapeutic intervention prevents some motor deficits by sparing the fine motor circuitry from secondary damage in both male and female mice.

\section{Materials and Methods}

\section{Lentiviral Gene Delivery}

Lentivirus containing pLenti-CMV-Luciferase or pLentiCMV-IL10 was produced as previously described [20]. Briefly, HEK-293FT cells (American Type Culture Collection, Manassas, VA) were transfected with lentiviral packing vectors and plasmids of interest in Opti-MEM (Life Technologies, Carlsbad, CA, \#31985-070) and Lipofectamine 2000 (Life Technologies, \#11668-019). After 48 h, the supernatant was collected and the virus was precipitated using PEG-It (Systems Biosciences, Palo Alto, CA, \#LV825A-1) and stored at $-80{ }^{\circ} \mathrm{C}$ until use. Prior to surgery, $4 \mathrm{e} 7 \mathrm{IFU}$ of virus was loaded onto each PLG scaffold, which were fabricated as previously described using the gas foaming technique to fuse PLG particles into a matrix [20, 38, 40]. Using the particulate leaching technique, 63-106 $\mu \mathrm{m} \mathrm{NaCl}$ served as sacrificial templates for pores to allow cell infiltration into the scaffold and a sugar mixture pulled into nine 150 250- $\mu \mathrm{m}$ diameter A-P parallel strands served as sacrificial templates for conduits to guide nerve growth $[36,41]$.

\section{Animals and Surgery}

All animal procedures conducted were approved by the Institutional Animal Care and Use Committee at the University of Michigan. For histology and ladder beam, mice 
used were 2-3-month-old C57BL/6 (Jackson Laboratories, Bar Harbor, ME, \#000664) at the time of injury. For optogenetics, mice used were 2-3-month-old Thy1-ChR2YFP line 18 (Jackson Laboratories \#007612) at the time of injury. Uninjured data is derived from age-, sex-, and genotype-matched controls at the time of sacrifice.

Mice were anesthetized under $2 \%$ isoflurane. The surgery site was then shaved and disinfected with iodine and $70 \%$ ethanol. Bupivacaine $(0.8 \mathrm{ml} / \mathrm{kg})$ was delivered locally to the incision site, and a C5 laminectomy was performed. Using a microfeather microscalpel (VWR, Radnor, PA, \#72045-15), a left lateral hemisection of 1-mm length (A-P) was performed at $\mathrm{C} 5$. The spinal tissue was excised and replaced with a lentivirus-loaded scaffold. Then the injury site was covered using GELFOAM (Pfizer, New York, NY), the overlying muscle was sutured using 5-0 Chromic Gut (Henry Schein, Melville, NY, \#101-8824), and the skin was stapled. Mice received buprenorphine $(0.1 \mathrm{mg} / \mathrm{kg})$ twice daily for 3 days, lactated ringer's solution fluid supplement $(5 \mathrm{ml} /$ $100 \mathrm{~g})$ daily for 5 days, and enrofloxacin $(2.5 \mathrm{mg} / \mathrm{kg})$ daily for 2 weeks.

\section{Behavioral Analysis}

C57BL/6 females only, due to persistent bladder issues in males, were trained to walk across a ladder beam consisting of $50+$ rungs prior to surgery as previously described [42]. After surgery, at 2 and 12 wpi, animals were placed on the ladder beam and coerced to walk across while being recorded on video. A minimum of 3 trials was completed for each animal at each time point. The videos were randomized among a total of four individuals such that each video was quantified by two counters who were blinded to the treatment condition. Then each video's quantifications were averaged between the two counters. A placement could be scored as (1) a complete placement with all toes facing forward and the palm on the rung, (2) a partial placement where the toes may be curled or the palm may not be centered though the limb is still weight-bearing, or (3) skipped rungs where the flanking rungs are placements. All others, such as misses or slips, were not counted. Examples can be seen in Fig. 1.

\section{Histological Analyses}

At 2 wpi, spinal tissues immediately caudal to the implant and arm muscles were extracted, flash frozen in isopentane on dry ice, then cryosectioned at $12 \mu \mathrm{m}$ in the transverse plane and $14 \mu \mathrm{m}$ in the longitudinal plane, respectively. Spinal and muscle tissues were stained following a standard immunohistochemistry protocol with fixation and without fixation in $4 \%$ paraformaldehyde, respectively. Antibodies used were mouse anti-NeuN (1:250, Millipore, Burlington, MA, \#MAB377) for mature neurons, chicken anti-NF200 (1:250, AVES Labs,

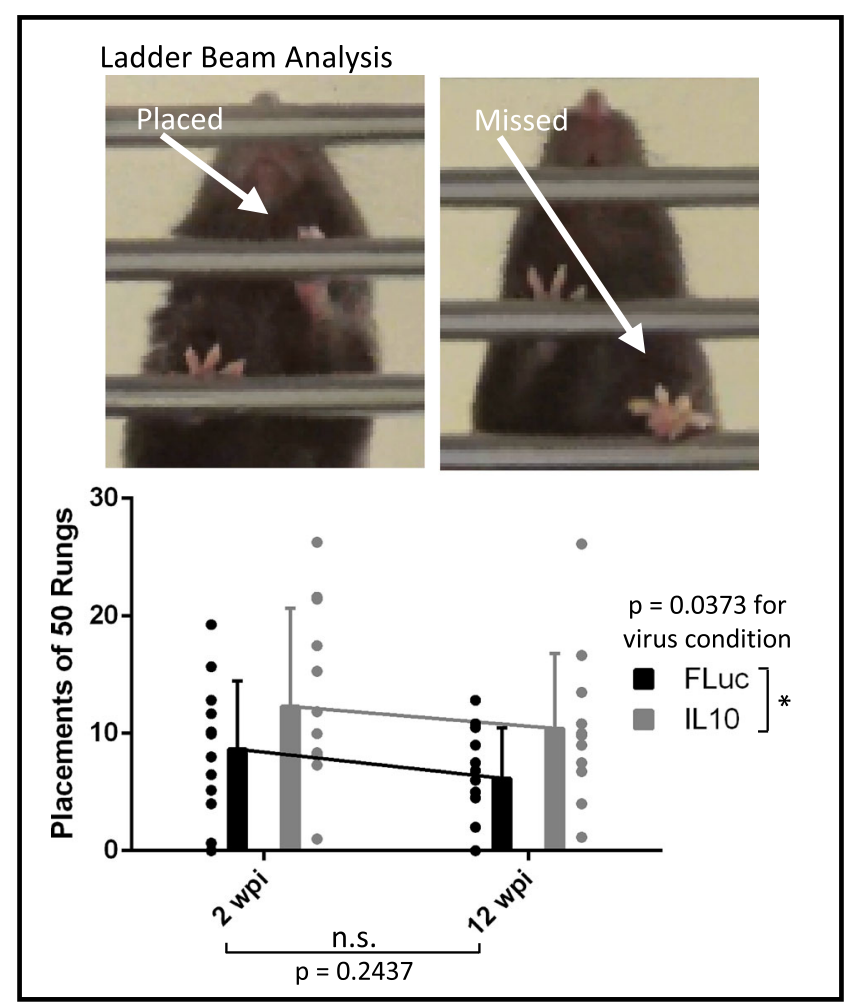

Fig. 1 Paw placements (top) and counts (bottom) show IL10-treated animals perform significantly better when compared to FLuc-treated animals. Error bars are \pm SD. $n=12-13$ animals/condition. Two-way ANOVA $* p<0.05$

Davis, CA, \#NFH) for neurofilaments, anti-bungarotoxin conjugated to Alexa Fluor 647 (1:500, Thermo Fisher, Waltham, MA, \#B35450) for motor endplates, and rabbit anti-GAP43 (1:100, Millipore, Burlington, MA, \#AB5220) for growth cones. Secondary antibodies were Alexa Fluor 647 donkey anti-mouse (1:1000, Jackson ImmunoResearch, West Grove, PA \#715-606-150), Alexa Fluor 488 goat anti-chicken (1:1000, Jackson ImmunoResearch, \#103-547-008), and Alexa Fluor 594 goat anti-rabbit (1:1000, Jackson ImmunoResearch, \#111-587-003).

Tissues were imaged using a Zeiss Axio Observer.Z1 microscope at 10X and processed in FIJI [43]. The analyzed particle function (0.5-1 circularity, exclude on edges) in FIJI was utilized to count cells of $100-250 \mu \mathrm{m}^{2}$ and 250 $1100 \mu \mathrm{m}^{2}$ within a region of interest (ROI) (i.e. ipsilateral or contralateral side) that had a threshold established by a single observer who was blinded to the experimental condition. A minimum total of 300 neurons across a minimum of 4 spinal cord sections from each spinal cord sample was quantified. For neurofilament (NFM) quantifications, a single observer, blinded to the experimental condition, outlined the ROI. Then, NFM-positive filaments within this ROI were quantified using a semi-automated counting program written in MATLAB as previously described [44]. Total motor endplate density, innervated motor endplate density, fraction of innervated motor 
endplates, and fraction of motor endplates with a growth cone nearby were quantified and averaged across two counters who were blinded to the experimental condition. If an NF200+ or GAP43+ axon colocalized or was $<1$ axon diameter away from a motor endplate, then it would be considered innervated or in the process of being reinnervated, respectively. A minimum total of 150 motor endplates across a minimum of 3 muscle sections from each muscle sample was quantified.

\section{Optogenetic Stimulation}

The spinal column of Thy1-ChR2-YFP mice anesthetized under 2\% isoflurane was exposed, and a C4-C6 laminectomy was performed from the dorsal side. The dura mater over $\mathrm{C} 4$ C6 was removed to allow insertion of a 1-mm-long LED probe (Plexon, Dallas, TX, \#OPT/FS-Flat-110/125-1L) into the left $\mathrm{C} 4$, midway between the lateral edge of the spinal cord and the midline. Optical stimulation was delivered using a PlexBright Controller to drive a 465-nm HELIOS headstage (Plexon, \#OPT/LED_Blue_HELIOS_LC_Kit) in a faraday cage. The optical stimulation parameters were 20 -ms pulse width at $1 \mathrm{~Hz}$ with a power of $0.04 \mathrm{~mW}$ for a minimum of 3 min per animal.

\section{EMG Data Acquisition and Processing}

Digital out and reference signals from the PlexBright Controller were sent to a headstage (Tucker-Davis Technologies, Alachua, FL, \#RA16AC) that was connected to a pre-amplifier (Tucker-Davis Technologies \#RA16PA), which converted the inputted electrical signal into an outputted optical signal. To gain access to the muscle, small incisions were made in the skin and needle electrodes (Natus Neurology, Pleasanton, CA, \#019-475400) were dually implanted into the left and right ATZ and FLX muscles. A single electrode in the left back ankle served as the reference signal. EMG and reference signals from the animal were connected to a second headstage and a second pre-amplifier. The optical outputs from each pre-amplifier were recorded by the same RX7 system (Tucker-Davis Technologies). A schematic for the equipment setup can be seen in Fig. 5(a) and an example EMG recording can be seen in Fig. 5(b).

Signals from both headstages were acquired at a synchronized rate of $24,414 \mathrm{~Hz}$ with bandpass frequencies of $2.2 \mathrm{~Hz}-$ $7.5 \mathrm{kHz}$. After acquisition, the data was converted to MATLAB ${ }^{\circledR}$ format using custom scripts. Next, the differential EMG electrode pairs were subtracted from each other and then band passed between 100 and $500 \mathrm{~Hz}$. To calculate the SNR for each muscle, a threshold was applied to the digital out signal representing the optical stimulation to determine the on and off times of the stimulation. Then, these times were used to calculate the root mean square values of the EMG signal during activation (stimulation on) and background (stimulation off). The ratio of the two values (on vs. off) was then used to calculate the SNR. To calculate the average P2P amplitude for each muscle, the maximum-to-minimum difference for each stimulation on time was determined, then averaged across all the stimulation on times for the length of that recording session.

\section{Results}

\section{Functional Recovery with IL10 Expression}

Initial studies analyzed the behavioral response following a penetrating injury to the cervical spinal cord. A left-sided hemisection to the cervical enlargement at $\mathrm{C} 5$ allowed isolation of SCI-induced motor deficits to only the left forelimb. The injury level, injury sidedness, and difficulty of placing a weight-bearing limb onto a ladder beam rung allowed quantification of movement that requires significant fine motor control. Previous reports show uninjured control animals averaged 50 placements [42]. Animals receiving a bridge with a lentivirus encoding IL10 averaged 12.3 and 10.4 placements at 2 and 12 wpi, respectively. Animals receiving a bridge with a lentivirus encoding FLuc averaged 8.7 and 6.2 left forelimb placements at 2 and 12 wpi, respectively. A two-way ANOVA indicates that IL10 expression significantly increased placements relative to FLuc expression $(p=0.037)$, while no differences in performance were observed over time $(p=0.24)$ or for interaction between time and virus condition $(p=0.87)$ (Fig. 1). These results are consistent with our previous reports using lentiviral IL10 in a hemisection thoracic model in which behavioral recovery was quantified using the Basso Mouse Scale (BMS) at 3, 14, 28, 42, 56, 70, and 84 days post injury [38], and in a hemisection cervical model in which behavioral recovery was quantified also using a ladder beam at $2,4,8$, and 12 wpi [21]. The ladder beam analysis was used here as it requires the use of specific muscle groups that are affected by the $\mathrm{C} 5$ injury. Furthermore, our hypothesis is that early tissue sparing due to decreased inflammation is the basis behind the preservation of function seen in animals treated with IL10. Thus, our subsequent histological and electrophysiological analyses focused on examining motor circuit integrity at 2 wpi when acute inflammation in untreated animals is transitioning to more chronic inflammation [45].

\section{Neuronal, Axonal, and NMJ Sparing with IL10}

Inflammation following trauma can result in the apoptosis of neurons, and we thus quantified peri-injury neuronal density. $\mathrm{NeuN}+$ neuron cell bodies range between 100 and $1100 \mu \mathrm{m}^{2}$, with LMNs having larger sizes $\left(250-1100 \mathrm{\mu m}^{2}\right)$ than interneurons $\left(100-250 \mu^{2}\right)$ [46]. Histological analyses of ipsilateral, peri-injury, caudal tissues at 2 wpi showed that animals 
treated with IL10 had significantly more interneurons and total neurons per section than animals treated with FLuc (Fig. 2(a-c)). Quantification of axon density in the ipsilateral side also indicated that animals treated with IL10 had significantly more axons than animals treated with FLuc (Fig. 2(d, e)). Since both the injured and contralateral side of FLuctreated animals trended towards decreased neuron densities for all sizes of neurons analyzed, we added age- and sexmatched, uninjured controls for all subsequent experiments.

Next, we analyzed sparing in the peripheral nervous system through the quantification of motor endplate density and NMJ innervation status. The NMJs were characterized as innervated (overlap of NF200+ neurofilament (NFM) and bungarotoxin (BGT)) (Fig. 3(a)), denervated (separation of NFM and BGT) (Fig. 3(b)), and reinnervated (overlap of BGT and growth-associated protein 43 (GAP43)) (Fig. 3(c)). For both females (Fig. 3(e, g, i, k)) and males (Fig. 4(b, d, $\mathrm{f}, \mathrm{h})$ ), a few significant differences were observed between IL10 and FLuc conditions in the contralateral tissue; meanwhile, males tended to have fewer significant differences between IL10 and FLuc conditions overall, thereby supporting the use of an age- and sex-matched uninjured control. Additionally, for both female and male FLX muscles, the analysis of the NMJs largely showed no significant difference between the experimental conditions and uninjured controls. The lack of difference between injury and control suggests that the injury was consistently rostral to where FLX muscle innervation began.

In the left ATZ of females, significant increases in motor endplate and innervated NMJ density per muscle area were observed in animals treated with either IL10 or FLuc relative to control uninjured animals (Fig. 3(d, f)). This result is consistent with previous reports showing that motor endplates will undergo fragmentation after a denervating event, resulting in an apparent increase in motor endplate density prior to the onset of muscle atrophy [47]. To account for fragmentation, we quantified the fraction of total motor endplates that are innervated and found that FLuc-treated animals had significantly decreased innervation when compared to that of control uninjured animals (Fig. 3(h)). Quantification of motor endplates colocalizing with growth cones showed that animals treated with FLuc also had the highest level of reinnervation (Fig. 3(j)). Since no difference in reinnervation or fraction of innervated motor endplates could be observed between IL10-treated and uninjured control animals, these data suggested IL10 spared more NMJs from denervation (Fig. 3(h, j)).

Overall, the differences observed for females (Fig. 3) were also seen in males (Fig. 4), yet to a lesser extent. The left ATZ in males treated with either IL10 or FLuc trended towards an increase in motor endplate and innervated NMJ densities (Fig. 4(a, c)). As with females, male mice treated with FLuc, but not IL10, had a significant decrease in their fraction of innervated motor endplates when compared to control uninjured animals (Fig. 4(e)). Animals treated with either IL10 or FLuc also trended towards an increase in their fraction of reinnervating motor endplates when compared to control uninjured animals (Fig. 4(g)). All $p$ values are reported in Supplementary Table 1.

\section{Sparing of Muscle Activation with IL10}

Next, we tested the hypothesis that NMJ differences identified by histology would influence muscle activation characteristics, which could be analyzed using electrophysiology. Denervation of an NMJ releases factors that can induce sprouting of nearby axons $[3,4]$. If a denervated NMJ becomes captured by a new motor unit, then stimulation of the adopting motor unit will result in an increased contractile force that is correlated with the amplitude of a compound action potential, which can be measured by an EMG. If a denervated NMJ remains orphaned, then stimulation of the motor unit that formerly owned that NMJ will show a decrease in the amplitude of its compound action potential [5-9]. Therefore, we quantified the compound action potential as a P2P amplitude after optogenetic stimulation of channelrhodopsin-positive $\mathrm{C} 4$ axons. Additionally, we quantified the SNR because background signals due to spontaneous fibrillations are a common characteristic of denervated muscles and can indicate damage to the motor circuitry independent of P2P changes.

No statistically significant differences in P2P and SNR values were observed between IL10-treated and uninjured control animals for both males and females, and for both the left ATZ and FLX muscles at 2 wpi (Fig. 5(c-h)), suggesting IL10 spared the motor circuitry from secondary damage. Meanwhile, females treated with FLuc had significantly lower left ATZ SNRs when compared to control uninjured animals (Fig. 5(d)), whereas males treated with FLuc had significantly lower left ATZ P2Ps and SNRs when compared to animals treated with IL10 (Fig. 5(g, h)). FLuc-treated males also had significantly lower left ATZ P2Ps when compared to uninjured control males (Fig. 5(g)). Together, these data show that the profound detrimental effects an SCI has on muscle activation, which can be identified in EMG recordings after optogenetic stimulation, can be partially alleviated using anti-inflammatory IL10 gene therapy. Finally, no trends or statistically significant differences could be observed in the contralateral side (Fig. $5(\mathrm{e}-\mathrm{j})$ ), which all had low values, suggesting that the optogenetic stimulation light was contained within the left C4. All $p$ values are reported in Supplementary Table 2 . 

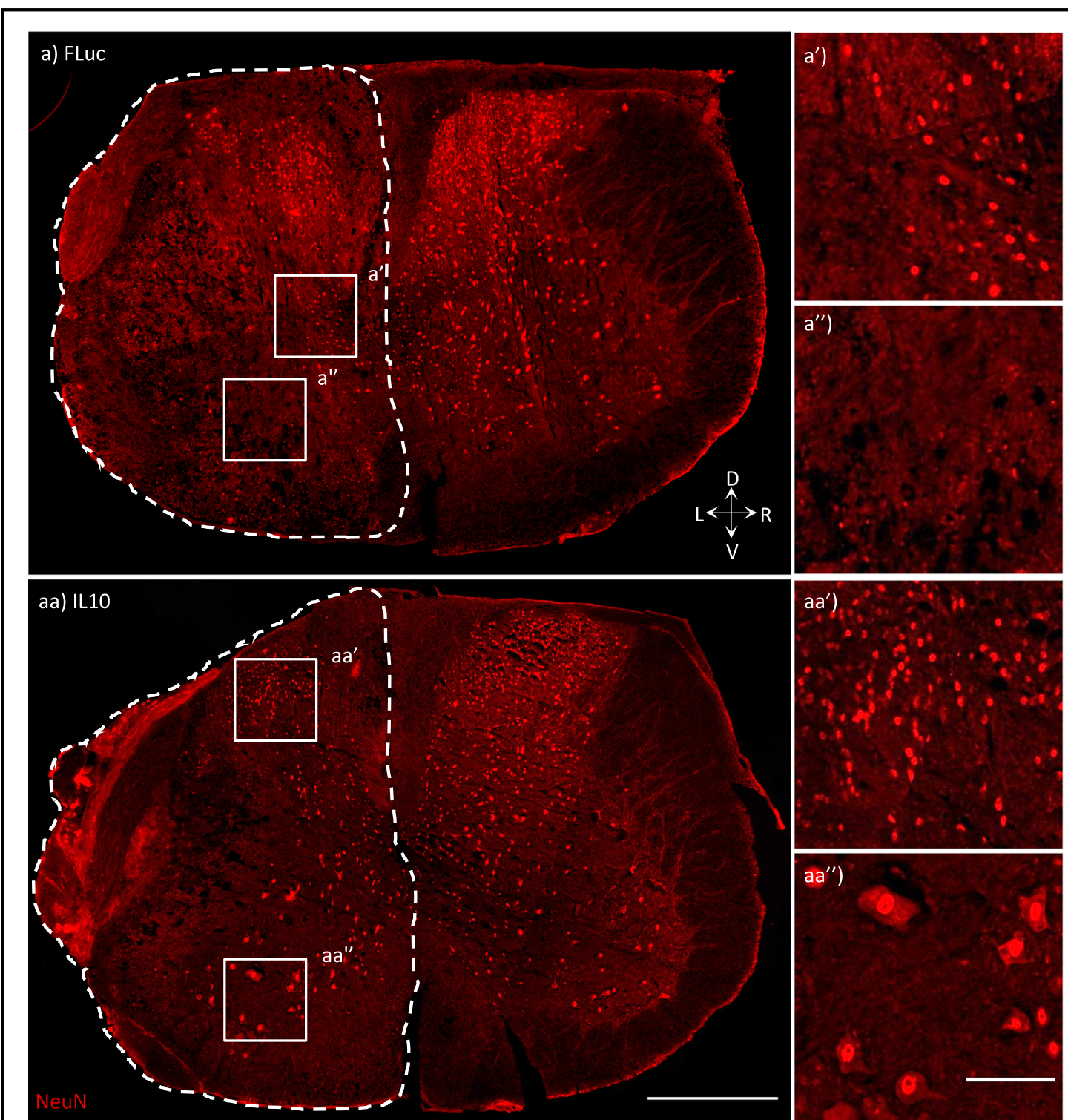

Left Side (2 wpi Females)

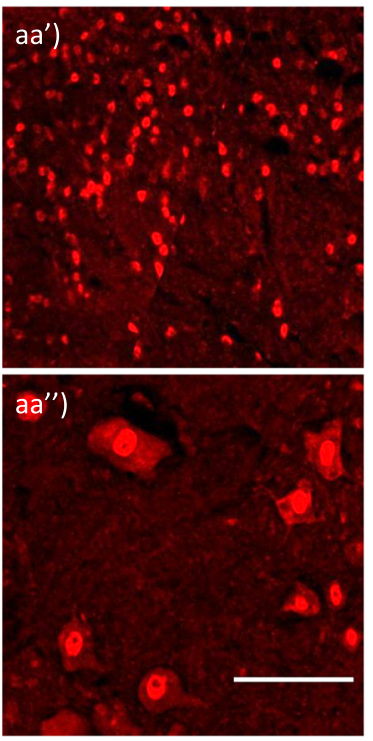

Right Side (2 wpi Females)

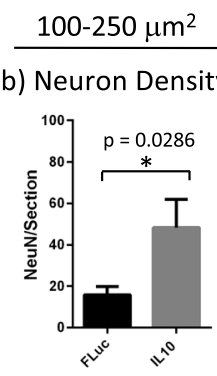
250-1100 $\mu \mathrm{m}^{2}$ $100-1100 \mu \mathrm{m}^{2}$

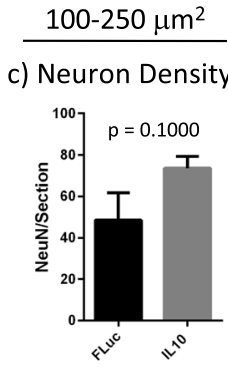
250-1100 $\mu \mathrm{m}^{2}$

$100-1100 \mu \mathrm{m}^{2}$
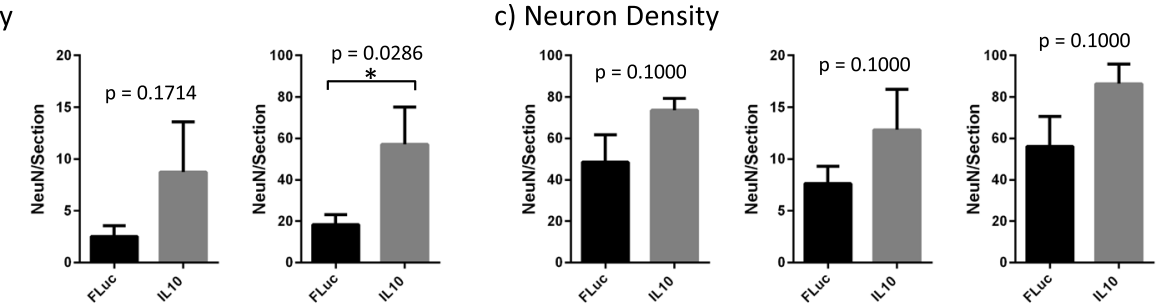

d) Axon Density
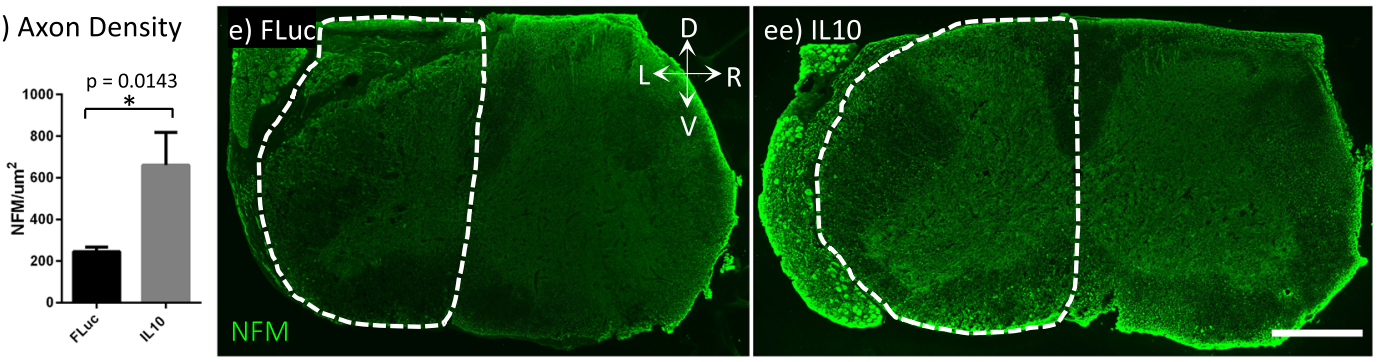
4Fig. 2 Tissue sections were stained for mature neurons (NeuN). Representative images of neurons in the sections for FLuc (a) and IL10 (a), 100-250 $\mathrm{m}^{2}$ size neurons (a', aa'), and 250-1100- $\mu \mathrm{m}^{2}$ size neurons (a", aa") are shown where the dotted lines represent the area quantified for the ipsilateral side. Quantifications of neurons normalized to each section for the left (b) and right (c) side are shown for each size range for 2 wpi females. Neurofilament (NFM) density was also quantified for just the ipsilateral side (d) from FLuc (e) and IL10 (ee) tissue sections stained for NFM where the dotted lines represent the area quantified. Error bars are \pm SEM. $n=4$ animals/condition. Mann-Whitney test ${ }^{*} p<0.05$. Scale bar $=500 \mu \mathrm{m}$ (a, aa, e, ee), $100 \mu \mathrm{m}$ (a', a", aa', aa")

\section{Discussion}

Our studies examined the potential for anti-inflammatory stimuli to enhance the fine motor circuitry and muscle activation in a cervical SCI model. The studies reported here validate that lentiviral IL10 gene therapy via a PLG implant into a left-sided, cervical SCI is associated with significantly improved performance of the left arm, as observed on a ladder beam. A number of groups have reported that IL10 treatment after a thoracic SCI can lead to intraspinal sparing of neurons, axons, and lesion size that is associated with improved locomotor function [23-29]. We have also previously demonstrated in a left-sided, thoracic SCI that IL10 can shift the inflammatory milieu towards a pro-regenerative phenotype, which was associated with significantly improved locomotor function $[37,38]$ and consistent with the results here in the cervical model. Thoracic injuries that employ behavioral tests such as the BMS for locomotion, which is controlled by central pattern generators (CPGs) that can be unilaterally activated to initiate bilateral locomotor behavior, may not fully capture the deficit from the unilateral injury. In contrast, cervical injuries are assessed through analysis of fine motor control and is controlled unilaterally [48]. Maintenance of fine motor control is also particularly relevant to humans, as we rely on forelimbs to a greater extent than rodents. Therefore, our studies provide further insight into IL10's therapeutic benefits on the fine motor circuitry, in contrast to previous behavioral tests on hindlimb function in which CPGs and reflexes play a larger role.

Additionally, we demonstrate that IL10 expression within the spinal cord impacts processes in the periphery. Specifically, examination of muscles innervated at the level of injury indicated that IL10 decreased NMJ denervation and prevented the pathological increase in motor unit and spontaneous fibrillations in those same muscles. In mice, the ATZ is innervated by LMNs whose cell bodies reside between $\mathrm{C} 2$ and C6 [39]. Within minutes after an injury, direct loss of the LMN cell body, such as removal via hemisection, can result in Wallerian degeneration of the axon and NMJ denervation $[49,50]$. This first denervating event is likely unavoidable because the majority of hemisected LMN cell bodies from C5 were innervating the left ATZ. Later, peri-injury LMNs can undergo apoptosis that peaks at 3 days post-injury in the highly inflammatory post-SCI environment, followed by axon degeneration and NMJ denervation $[11,51$, 52]. This second denervating event is the target of our anti-inflammatory treatment. After a denervating event, motor endplates will undergo fragmentation [47, 53, 54]. The cumulative effects of direct removal of the LMN cell body and secondary damage due to inflammation can be seen most profoundly in the 2 wpi left ATZ of animals treated with FLuc, which had a significant increase in motor endplate density that reflects fragmentation and a significant decrease in the fraction of total motor endplates that are innervated when compared to uninjured control animals. Meanwhile, although the 2 wpi left ATZ of IL10-treated animals also showed significant motor endplate fragmentation, the fraction of total motor endplates that are innervated remained comparable to that of uninjured controls. Since animals treated with IL10 also had far fewer growth cones than animals treated with FLuc, suggesting a decreased need for reinnervation, together these data indicate a decrease in injury severity that is likely due to our anti-inflammatory treatment. We therefore identify IL10-induced sparing of NMJs in the left ATZ as a major contributor to improved fine motor control after a cervical SCI.

We report that IL10 treatment was effective in both male and female mice, though with some differences. All previous studies for delivery of IL10 used female rodents [21-27, 29, 37], except for one that did not indicate the sex of their animals [28]. Since differences in the neuroinflammatory response and locomotor recovery between male and female mice have been identified [55], IL10 treatment could potentially have disparate effects in each sex. For all the electrophysiological measures, no difference could be found between uninjured and IL10treated animals, further supporting therapeutic benefits of IL10 in both sexes. Interestingly, control injury significantly reduced the P2P of the left ATZ, reflecting a decrease in motor unit size due to injury, in males but not in females. Since we found females treated with FLuc had significantly increased reinnervation, then reestablishment of a normal motor unit size might account for the apparently normal P2P seen in the left ATZ. Whether or not females have more or accelerated reinnervation relative to males remains to be determined. We do not anticipate that the normal P2P of the ATZ in females treated with FLuc reflects a lack of pathological outcomes, because increased pathological fibrillations explain why the SNR in females was decreased despite no observable reduction 

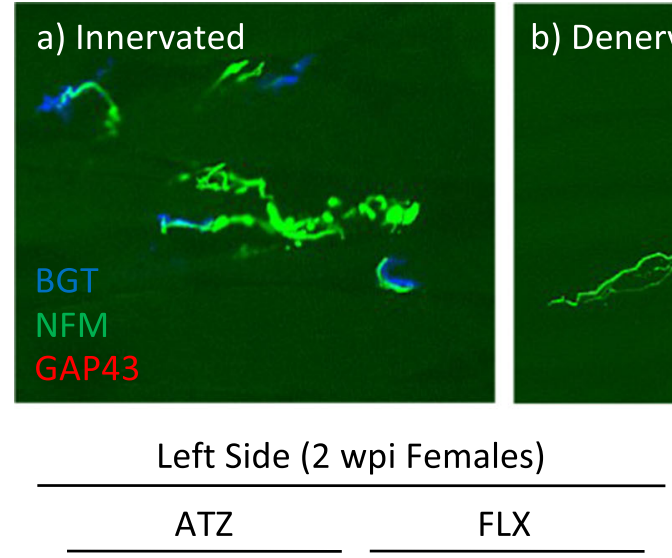

d) Motor Endplate Density
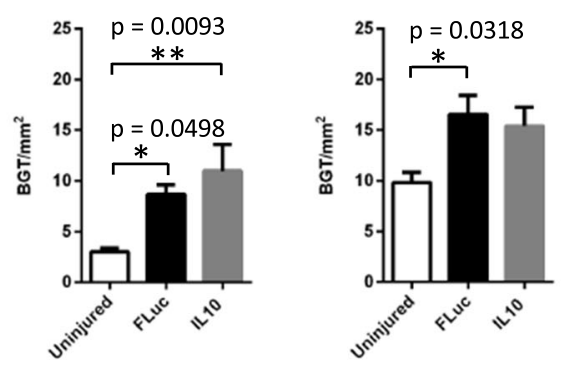

f) Innervated NMJ Density
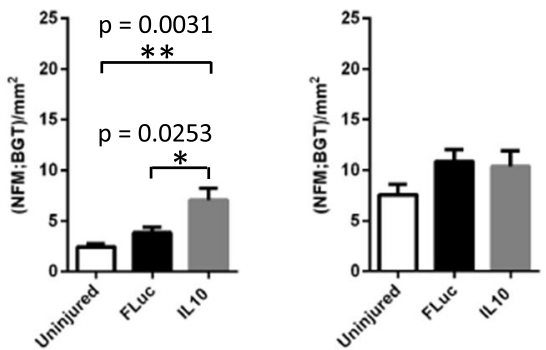

h) Fraction of Innervated Motor Endplates
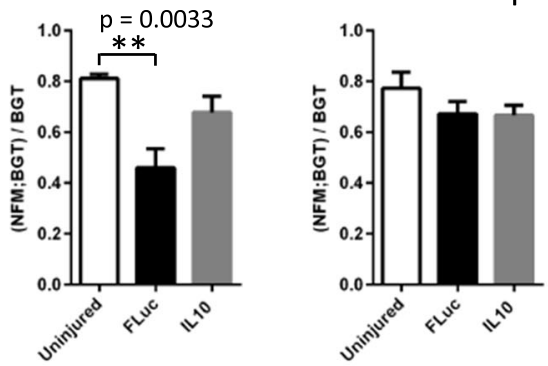

j) Fraction of Reinnervating Motor Endplates
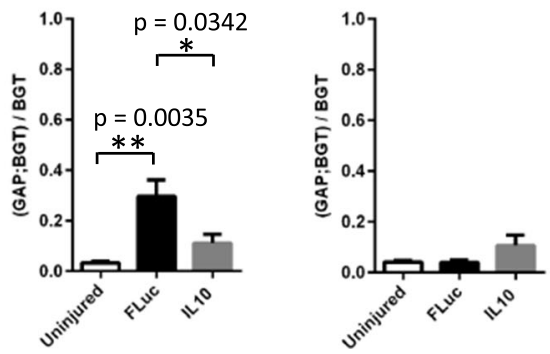

Right Side (2 wpi Females)

ATZ

e) Motor Endplate Density
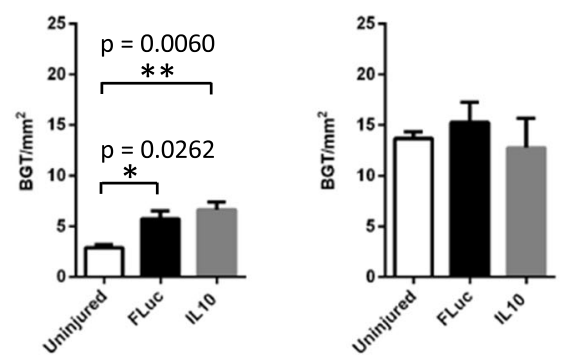

g) Innervated NMJ Density
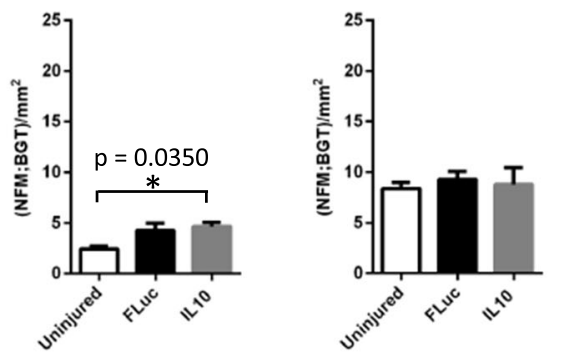

i) Fraction of Innervated Motor Endplates
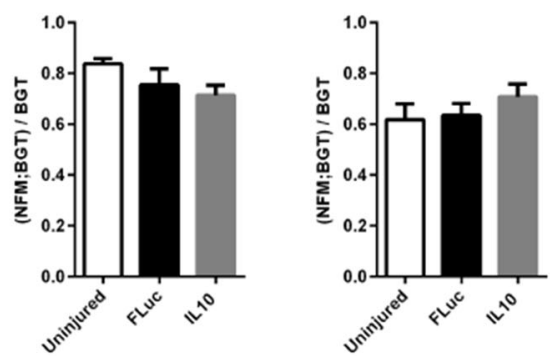

k) Fraction of Reinnervating Motor Endplates
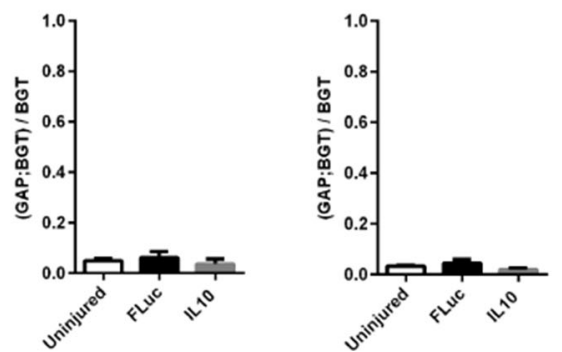
Fig. 3 Tissue sections were stained for bungarotoxin (BGT) for motor endplates, neurofilament (NFM) for axons, and growth-associated protein 43 (GAP43) for growth cones. Representative images of NMJs that are (a) innervated, (b) denervated, or being (c) reinnervated are shown. Quantifications of motor endplate density normalized to muscle area (d, e), innervated NMJ density normalized to muscle area (f, g), fraction of total motor endplates that are innervated $(\mathrm{h}, \mathrm{i})$, and fraction of total motor endplates that are being reinnervated (j, $\mathrm{k}$ ) are shown for 2 wpi females. Error bars are \pm SEM. $n=5-6$ animals/condition. One-way ANOVA with Tukey post hoc $* p<0.05, * * p<0.01$. Scale bar $=50 \mu \mathrm{m}$

in the P2P values. Additionally, while the electrophysiological effects of IL10 in males were clear, males showed fewer histological differences between conditions. Since all experiments were carried out in C57/BL6 background mice, and increased testosterone has been linked with decreased functional outcomes [56], we suspect that the observed sex differences reflect mechanistic differences in the injury response.

While tissue sparing is associated with preservation of function as measured by behavior and by optical stimulation at 2 wpi, no additional behavioral improvements were observed at 12 wpi. Hemisected intraspinal reflex and locomotor circuits will inevitably result in the loss of some synchronized muscle activation that is necessary for normal gait to occur. Additionally, increases in motor unit size due to reinnervation will decrease an animal's control over their contractile force, such that the ratio of cognitive effort necessary to elicit a specific force will be
Fig. 4 Quantifications of motor endplate density normalized to muscle area ( $a, b)$, innervated NMJ density normalized to muscle area (c, d), fraction of total motor endplates that are innervated (e, f), and fraction of total motor endplates that are being reinnervated $(\mathrm{g}, \mathrm{h})$ are shown for 2 wpi males. Error bars are \pm SEM. $n=5-6$ animals/ condition. One-way ANOVA with Tukey post hoc $* p<0.05$

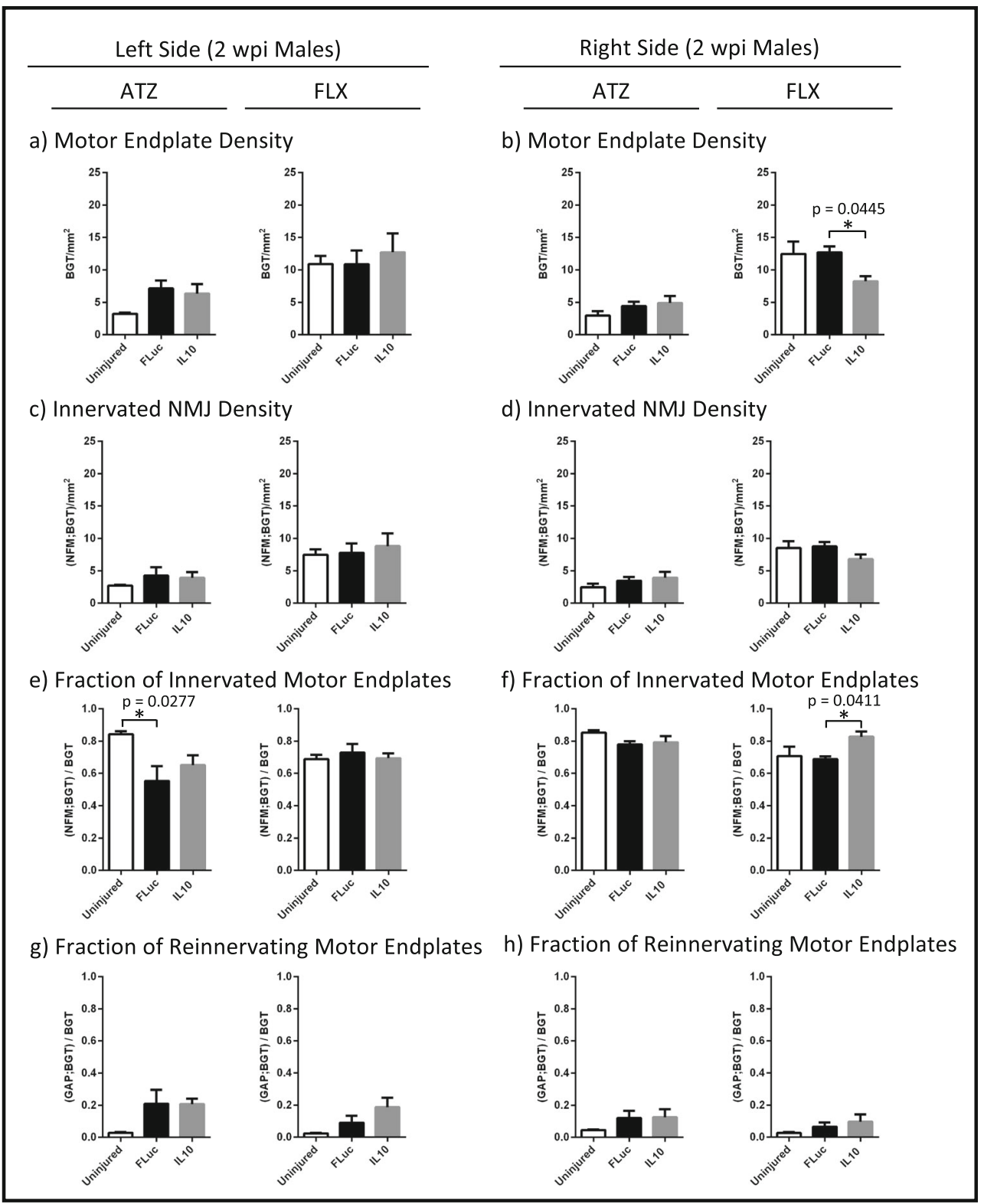




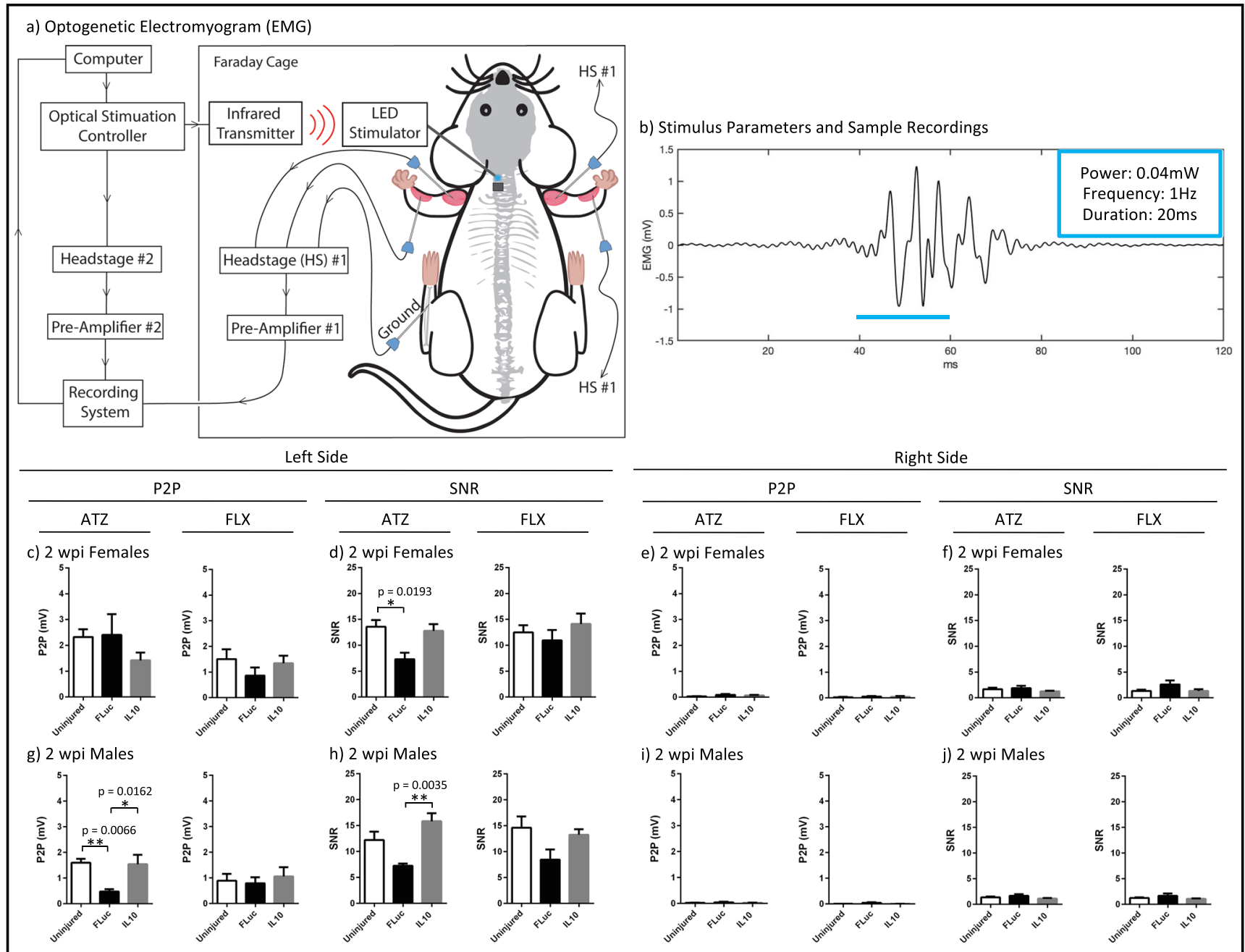

Fig. 5 Schematic of the equipment setup (a) for optogenetically evoked electromyogram recordings is shown, as well as the stimulus parameters (20 ms pulses, $0.04 \mathrm{~mW}, 3 \mathrm{~min}, 1 \mathrm{~Hz}$ ) and a representative EMG trace (b). The peak-to-peak (c, e, g, i) and signal-to-noise ratios $(\mathrm{d}, \mathrm{f}, \mathrm{h}, \mathrm{j})$ for $2 \mathrm{wpi}$

shifted during fine motor movements [57]. If a muscle or neuron becomes inappropriately reinnervated after injury, the resulting synkinesis can further disrupt the synchronized muscle activation that is necessary for coordinated movement. Therefore, though sparing the motor circuitry by decreasing secondary damage due to inflammation can prevent some of the motor deficits that often results from an SCI, future studies might need to focus on the specificity of circuit reconnection in order for proper behavioral recovery to occur.

\section{Conclusions}

This study utilizes a left-sided, cervical level SCI to demonstrate that lentiviral IL10 can prevent some motor deficits from forming in the left arm. Intraspinal expression of IL10 can preserve NMJ innervation in the peripheral nervous

females (c, d, e, f) and 2 wpi males (g, h, i, j) were quantified for the ATZ and FLX muscles. Error bars are \pm SEM. $n=4-8$ animals/condition. Oneway ANOVA with Tukey post hoc $* p<0.05, * * p<0.01$

system. Using optogenetic stimulation of $\mathrm{C} 4$ axons that originate from channelrhodopsin positive layer $\mathrm{V}$ neurons, we were able to examine muscle activation due solely to the fine motor circuitry and demonstrate that IL10 treatment can prevent pathological EMG signals in injury-affected muscles. Furthermore, these findings were mostly consistent in both male and female mice indicating potential applicability for both sexes. Collectively, our results indicate that early immunomodulatory intervention can yield long-term benefits.

Acknowledgements The authors would like to thank Drs. Eva Feldman, Jonghyuck Park, Dominique Smith, and Courtney Dumont for their mentorship and guidance, as well as John Hayes and Rohit Maramraju for contributing their technical expertise. Funding for this work was provided by the National Institutes of Health (R01AI148076), the National Institute of Neurological Disorders and Stroke (U01NS094375 and UF1NS107659 for P.R.P.), the Office of the Director National institutes of Health (OT2OD024907 for P.R.P.), the National Science Foundation (1707316 for P.R.P. and Graduate Research Fellowship for J.Y.C.), and the University of Michigan (Rackham Merit Fellowship for J.Y.C.). 


\section{References}

1. Harel NY, Strittmatter SM. Can regenerating axons recapitulate developmental guidance during recovery from spinal cord injury? Nat Rev Neurosci 2008;7(8):603-16.

2. Wu P, Chawla A, Spinner RJ, Yu C, Yaszemski MJ, Windebank $\mathrm{AJ}$, et al. Key changes in denervated muscles and their impact on regeneration and reinnervation. Neural Regen Res 2014;9(20): 1796-809.

3. Kuffler DP. Regeneration of muscle axons in the frog is directed by diffusible factors from denervated muscle and nerve tubes. J Comp Neurol 1989;281(3):416-25.

4. Hepple RT, Rice CL. Innervation and neuromuscular control in ageing skeletal muscle. J Physiol 2016;594(8):1965-78.

5. Rowan SL, Rygiel K, Purves-Smith FM, Solbak NM, Turnbull DM, Hepple RT. Denervation causes fiber atrophy and myosin heavy chain co-expression in senescent skeletal muscle. PLoS One 2012;7(1):e29082.

6. Spector SA. Trophic effects on the contractile and histochemical properties of rat soleus muscle. J Neurosci 1985;5(8):2189-96.

7. Carlson BM, Billington L, Fauklner J. Studies on the regenerative recovery of long-term denervated muscle in rats. Restor Neurol Neurosci 1996;10(2):77-84.

8. Kalliainen LK, Jejurikar SS, Liang LW, Urbanchek MG, Kuzon Jr. WM. A specific force deficit exists in skeletal muscle after partial denervation. Muscle Nerve 2002;25(1):31-8.

9. Dow DE, Cederna PS, Hassett CA, Kostrominova TY, Faulkner JA, Dennis RG. Number of contractions to maintain mass and force of a denervated rat muscle. Muscle Nerve 2004;30(1):77-86.

10. David S, López-Vales R, Yong VW. Chapter 30 - Harmful and beneficial effects of inflammation after spinal cord injury: potential therapeutic implications. Handb Clin Neurol 2012;109:485-502.

11. Zhang N, Yin Y, Xu S-J, Wu Y-P, Chen W-S. Inflammation \& apoptosis in spinal cord injury. Indian J Med Res 2012;135 (3): 287-96.

12. Vajn K, Plunkett JA, Tapanes-Castillo A, Oudega M. Axonal regeneration after spinal cord injury in zebrafish and mammals: differences, similarities, translation. Neurosci Bull 2013;29(4): 40210.

13. Ley K. M1 means kill; m2 means heal. J Immunol 2017;199(7): 2191-3.

14. Zhang B, Bailey WM, Braun KJ, Gensel JC. Age decreases macrophage IL-10 expression: Implications for functional recovery and tissue repair in spinal cord injury. Exp Neurol 2015;273:83-91.

15. Lopes RL, Borges TJ, Zanin RF, Bonorino C. IL-10 is required for polarization of macrophages to M2-like phenotype by mycobacterial DnaK (heat shock protein 70). Cytokine. 2016;85:123-9.

16. Thompson CD, Zurko JC, Hanna BF, Hellenbrand DJ, Hanna A. The therapeutic role of interleukin-10 after spinal cord injury. $\mathrm{J}$ Neurotrauma 2013;30(15):1311-24.

17. Boehler RM, Kuo R, Shin S, Goodman AG, Pilecki M, Leonard J, et al. Lentivirus delivery of IL-10 to promote and sustain macrophage polarization towards an anti-inflammatory phenotype. Biotechnol Bioeng 2014;111(6):1210-21.

18. Genovese T, Esposito E, Mazzon E, Di Paola R, Caminiti R, Bramanti $\mathrm{P}$, et al. Absence of endogenous interleukin-10 enhances secondary inflammatory process after spinal cord compression injury in mice. J Neurochem 2009;108(6):1360-72.

19. Zhou Z, Peng X, Insolera R, Fink D, Mata M. Interleukin-10 provides direct trophic support to neurons. J Neurochem 2009;110(5): 1617-27.

20. Park J, Decker JT, Smith DR, Cummings BJ, Anderson AJ, Shea LD. Reducing inflammation through delivery of lentivirus encoding for anti-inflammatory cytokines attenuates neuropathic pain after spinal cord injury. J Control Release 2018;290:88-101.
21. Smith DR, Dumont CM, Park J, Ciciriello A, Guo A, Tatineni R, et al. Polycistronic delivery of IL-10 and NT-3 promotes oligodendrocyte myelination and functional recovery in a mouse spinal cord injury model. Tissue Eng Part A. 2020;26.

22. Brewer K, Bethea J, Yezierski R. Neuroprotective effects of interleukin-10 following excitotoxic spinal cord injury. Exp Neurol 1999;159(2):484-93.

23. Takami T, Oudega M, Bethea JR, Wood P, Kleitman N, Bunge M. Methylprednisolone and interleukin-10 reduce gray matter damage in the contused fischer rat thoracic spinal cord but do not improve functional outcome. J Neurotrauma 2002;19(5):653-66.

24. Ishii H, Tanabe S, Ueno M, Kubo T, Kayama H, Serada S, et al. ifn$\gamma$-dependent secretion of IL-10 from Th1 cells and microglia/ macrophages contributes to functional recovery after spinal cord injury. Cell Death Dis. 2013;4(7):e710.

25. Hellenbrand DJ, Reichl K, Travis B, Filipp M, Khalil A, Pulito D, et al. Sustained interleukin-10 delivery reduces inflammation and improves motor function after spinal cord injury. J Neuroinflammation 2019;16(1):93.

26. Zhou Z, Peng X, Insolera R, Fink D, Mata M. IL-10 promotes neuronal survival following spinal cord injury. Exp Neurol 2009;220(1):183-90.

27. Oruckaptan H, Ozisik P, Atilla P, Tuncel M, Kilinc K, Geyik P, et al. Systemic administration of interleukin-10 attenuates early ischemic response following spinal cord ischemia reperfusion injury in rats. J Surg Res 2009;155(2):345-56.

28. Jackson C, Messinger J, Peduzzi J, Ansardi D, Morrow C. Enhanced functional recovery from spinal cord injury following intrathecal or intramuscular administration of poliovirus replicons encoding IL-10. Virology 2005;336(2):173-83.

29. Bethea JR, Nagashima H, Acosta M, Briceno C, Gomez F, Marcillo A, et al. Systemically administered interleukin-10 reduces tumor necrosis factor-alpha production and significantly improves functional recovery following traumatic spinal cord injury in rats. $\mathrm{J}$ Neurotrauma 1999;16(10):851-63.

30. Koushki D, Latifi S, Javidan AN, Matin M. Efficacy of some nonconventional herbal medications (sulforaphane, tanshinone IIA, and tetramethylpyrazine) in inducing neuroprotection in comparison with interleukin-10 after spinal cord injury: a meta-analysis. J Spinal Cord Med 2015;38(1):13-22.

31. Spinal Cord Injury: Facts and Figures at a Glance. In: National Spinal Cord Injury Statistics Center UoA, Birmingham [online]. Available at: https://www.nscisc.uab.edu/Public/Facts\%20and\% 20Figures\%202020.pdf. Accessed June 5, 2020.

32. Mapplebeck J, Beggs S, Salter M. Sex differences in pain: a tale of two immune cells. Pain 2016;157:S2-6.

33. Rosen S, Ham B, Mogil J. Sex differences in neuroimmunity and pain. J Neurosci Res 2017;95:500-8.

34. Elkabes S, Nicot AB. Sex steroids and neuroprotection in spinal cord injury: A review of preclinical investigations. Exp Neurol 2014;259:28-37.

35. Singh A, Tetreault L, Kalsi-Ryan S, Nouri A, Fehlings MG. Global prevalence and incidence of traumatic spinal cord injury. Clin Epidemiol 2014;6:309-31.

36. Thomas AM, Shea LD. Polysaccharide-modified scaffolds for controlled lentivirus delivery in vitro and after spinal cord injury. $\mathrm{J}$ Control Release 2013;170(3):421-9.

37. Margul DJ, Park J, Boehler RM, Smith DR, Johnson MA, McCreedy DA, et al. Reducing neuroinflammation by delivery of IL-10 encoding lentivirus from multiple-channel bridges. Bioeng Transl Med 2016;1(2):136-48.

38. Park J, Decker JT, Margul DJ, Smith DR, Cummings BJ, Anderson AJ, et al. Local Immunomodulation with Anti-inflammatory Cytokine-Encoding Lentivirus Enhances Functional Recovery after Spinal Cord Injury. Mol Ther 2018;26(7):1756-70. 
39. Tosolini AP, Mohan R, Morris R. Targeting the Full Length of the Motor End Plate Regions in the Mouse Forelimb Increases the Uptake of Fluoro-Gold into Corresponding Spinal Cord Motor Neurons. Front Neurol 2013;4.

40. Smith DR, Margul DJ, Dumont CM, Carlson MA, Munsell MK, Johnson $\mathrm{M}$, et al. Combinatorial lentiviral gene delivery of prooligodendrogenic factors for improving myelination of regenerating axons after spinal cord injury. Biotechnol Bioeng 2019;116(1):15567.

41. Thomas AM, Kubilius MB, Holland SJ, Seidlits SK, Boehler RM, Anderson AJ, et al. Channel density and porosity of degradable bridging scaffolds on axon growth after spinal injury. Biomaterials. 2013;34(9):2213-20.

42. Cummings BJ, Engesser-Cesar C, Anderson AJ. Adaptation of a ladder beam walking task to assess locomotor recovery in mice following spinal cord injury. Behav Brain Res 2008;117(2):232-41.

43. Schindelin J, Arganda-Carreras I, Frise E, Kaynig V, Longair M, Pietzsch T, et al. Fiji: an open-source platform for biological-image analysis. Nat Methods 2012;9(7):676-82.

44. McCreedy DA, Margul DJ, Seidlits SK, Antane JT, Thomas RJ, Sissman GM, et al. Semi-Automated Counting of Axon Regeneration in Poly (Lactide Co-Glycolide) Spinal Cord Bridges. J Neurosci Methods 2016;263:15-22.

45. Sroga JM, Jones TB, Kigerl KA, McGaughy VM, Popovich PG. Rats and mice exhibit distinct inflammatory reactions after spinal cord injury. J Comp Neurol 2003;462(2):223-40.

46. Wu L, Cheng W, Shen C. Targeted depletion of TDP-43 expression in the spinal cord motor neurons leads to the development of amyotrophic lateral sclerosis-like phenotypes in mice. J Biol 2012;287(33):27335-44

47. Labovitz S, Robbins N, Fahim M. Endplate topography of denervated and disused rat neuromuscular junctions: comparison by scanning and light microscopy. Neurosci. 1984;11(4):963-71.

48. Causes of a spinal cord injury. In: Christopher \& Dana Reeve Foundation [online]. Available at: https://www.christopherreeve. org/living-with-paralysis/health/causes-of-paralysis/spinal-cordinjury. Accessed June 5, 2020.

49. Kerschensteiner M, Schwab ME, Lichtman J, Misgeld T. In vivo imaging of axonal degeneration and regeneration in the injured spinal cord. Nat Med 2005;11(5):572-7.

50. Lingor P, Koch JC, Tönges L, Bähr M. Axonal degeneration as a therapeutic target in the CNS. Cell Tissue Res 2012;349(1):289311.

51. Hassannejad Z, Zadegan SA, Shakouri-Motlagh A, Mokhatab M, Rezvan M, Sharif-Alhoseini M, et al. The fate of neurons after traumatic spinal cord injury in rats: A systematic review. Iran J Basic Med Sci 2018;21(6):546-57.

52. Yong C, Arnold P, Zoubine M, Citron B, Watanabe I, Berman N, et al. Apoptosis in cellular compartments of rat spinal cord after severe contusion injury. J Neurotrauma 1998;15(7):459-72.

53. Apel PJ, Alton T, Northam C, Ma J, Callahan M, Sonntag WE, et al. How age impairs the response of the neuromuscular junction to nerve transection and repair. J Orthop Res 2009;27(3):385-93.

54. Wilson M, Deschenes M. The neuromuscular junction: anatomical features and adaptations to various forms of increased, or decreased neuromuscular activity. Int J Neurosci 2005;115(6):803-28.

55. Farooque M, Suo Z, Arnold P, Wulser M, Chou C-T, Vancura R, et al. Gender-related differences in recovery of locomotor function after spinal cord injury in mice. Spinal Cord 2006;44:182-7.

56. Hauben E, Mizrahi T, Agranov E, Schwartz M. Sexual dimorphism in the spontaneous recovery from spinal cord injury: a gender gap in beneficial autoimmunity? Eur J Neurosci 2002;16(9)1731-40.

57. Thomas CK, Zijdewind I. Fatigue of muscles weakened by death of motoneurons. Muscle Nerve 2005;33(1):21-41.

Publisher's Note Springer Nature remains neutral with regard to jurisdictional claims in published maps and institutional affiliations. 